\title{
DES is not a Group
}

\author{
Keith W. Campbell and Michael J. Wiener \\ Bell-Northern Research, P.O. Box 3511 Station C, Ottawa, Ontario, Canada, K1Y 4H7
}

\begin{abstract}
We prove tbat the set of DES permutations (encryption and decryption for each DES key) is not closed under functional composition. This implies that, in general, multiple DES-encryption is not equivalent to single DES-encryption, and that DES is not susceptible to a particular known-plaintext attack which requires, on average, $2^{28}$ steps. We also show that the size of the subgroup generated by the set of DES permutations is greater than $10^{2499}$, which is too large for potential attacks on DES which would exploit a small subgroup.
\end{abstract}

\section{Introduction}

The Data Encryption Standard (DES) [3] defines a set of permutations on messages from the set $M=\{0,1\}^{64}$. The permutations consist of encryption and decryption with keys from the set $K=\{0,1\}^{56}$. Let $E_{k}: M \rightarrow M$ denote the encryption permutation for key $k$, and let $E_{k}^{-1}$ be the corresponding decryption permutation. If the set of DES permutations were closed under functional composition, then for any two permutations $t$ and $u$, there would exist some other permutation $v$ such that $u(t(m))=v(m)$ for all messages $m \in M$.

The question of whether the set of DES permutations is closed under functional composition is an important one because closure would imply that there exists a knownplaintext attack on DES that requires, on average, $2^{28}$ steps [4]. Furthermore, multiple encryption would be susceptible to the same attack because multiple encryption would be equivalent to single encryption.

Kaliski, Rivest, and Sherman developed novel cycling tests which gave evidence that the set of DES permutations is not closed [4]. However, their work relied upon randomness assumptions about either DES itself or a pseudo-random function $\rho: M \rightarrow K$ which was used in cycling experiments. Because of the randomness assumptions, it is difficult to use the results of their cycling tests to make any claims about the probability that DES is not closed.

We have developed our own DES cycling experiments which provide evidence that DES is not closed; this evidence does not rely upon randomness assumptions. Our cycling experiments are similar to those of Quisquater and Delescaille for finding DES collisions $[7,8]$. Other recent related work is the switching ciosure tests of Morita, Ohta, and Miyaguchi [6]. 
Don Coppersmith has developed an approach to finding a lower bound on the size of the subgroup generated by the DES permutations [1]. He has shown this lower bound to be greater than the number of DES permutations, providing conclusive proof that DES is not closed.

Section 2 contains the new probabilistic argument against closure which relies upon the ability to find a set of four keys which quadruple-encrypt a particular plaintext message to a particular ciphertext message. Finding such four-key mappings can be done with an approach similar to finding DES collisions. In Section 3, we review previous work in collision finding and build up to the new method of finding four-key mappings. Section 4 contains further details on our experiments. In Section 5, we describe Don Coppersmith's approach to obtaining a lower bound on the size of the subgroup generated by the DES permutations, thereby proving that DES is not closed. We also discuss our results based on his approach.

\section{Strong Evidence Against Closure}

We begin with the hypothesis that the set of DES permutations is closed and search for a contradiction. Let $S_{p}$ be the set of messages that can result from encrypting or decrypting a particular message $p$ with any DES key. Because there are $2^{56}$ keys, $S_{p}$ contains at most $2^{57}$ messages. From the hypothesis, $S_{p}$ is also the set of all possible messages which can result when multiple permutations are applied to $p$. If a message $c \in M$ is selected at random, the probability that $c \in S_{p}$ is at most $2^{57 / 2} / 2^{64}=2^{-7}$. We selected 50 messages at random (by coin tossing), and for each random message $c$, we searched for a set of permutations which map $p$ to $c$ using $p=0$ in each case. In all 50 cases we found a set of four DES keys $i, j, k$, and $l$ such that $E_{l}\left(E_{k}\left(E_{j}\left(E_{i}(p)\right)\right)\right)=c$ (see Appendix). Therefore, $c \in S_{p}$ and the probability of this event occurring 50 times is at most $\left(2^{-7}\right)^{50}=2^{-350}$. Because this is an extremely unlikely occurence, we must conclude that the original hypothesis is incorrect and the set of DES permutations is (almost certainly) not closed under functional composition.

The argument above does not rely upon any assumptions about the randomness of DES or any other function; the fact that four keys exist which map $p$ to $c$ for each randomly selected message $\mathrm{c}$ is sufficient to draw the conclusion. However, the method used to find the four keys in each case does rely upon randomness assumptions.

\section{Collision Finding}

The method used to find four keys which map one message to another is similar to the approach taken by Quisquater and Delescaille in finding DES collisions ${ }^{1}$ [7]. In both cases a function $f: M \rightarrow M$ and an initial message $x_{0}$ are chosen which define the sequence $x_{i+1}=f\left(x_{i}\right)$ for $i=0,1, \ldots$. Because $M$ is finite, this sequence must eventually fall into a cycle. Unless $x_{0}$ is in the cycle, the sequence consists of a leader flowing into a cycle. The algorithms described by Sedgewick, Szymanski, and Yao [9] can be used to find the leader

I We bave a DES collision when $E_{i}(m)=E_{j}(m)$ for some $m \in M$, and $i, j \in K, i \neq j$. 
length $\lambda$ and the cycle length $\mu$. If $\lambda \neq 0$, this leads directly to finding a collision in $f$ (i.e., $a, b \in M$ such that $f(a)=f(b), a \neq b$, see Figure 1$)$.

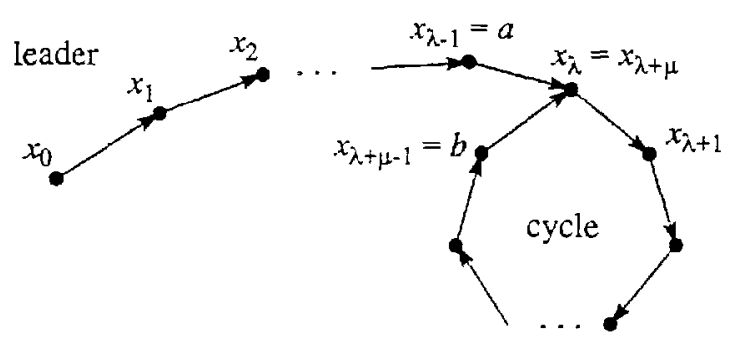

Figure 1. Leader and Cycle in a Sequence

\section{DES Collisions}

To find DES collisions, Quisquater and Delescaille used the function $f(x)=E_{g(x)}(m)$, where $g: M \rightarrow K$ takes a message and produces a key for DES encryption, and $m$ is a fixed message. In this case, a collision in $f$ is not necessarily a DES collision; if $f(a)=f(b)$, $a \neq b$, but $g(a)=g(b)$, then we have found a pseudo-collision where the keys are the same. Because there are fewer keys than messages, there can be at most $|K|$ distinct outputs from $f$. Assuming that DES is random and a suitable function $g$ is selected, the probability of a collision in $f$ leading to a DES collision is about $|K| /|M|=2^{-8}$, and the expected time required to find a collision in $f$ is on the order of $\sqrt{|K|}=2^{28}$. Thus, the overall work factor in repeating this procedure until a DES collision is found is about $2^{28 / 2} / 2^{-8}=2^{36}$. This can be reduced somewhat using the method of distinguished points [7].

\section{Two-Key Mapping}

The method of finding DES collisions above was extended by Quisquater and Delescaille to find pairs of keys which double-encrypt a particular plaintext $p$ to produce a particular ciphertext $c$ [8]. In this case, collisions were found between two functions $f_{1}(x)=E_{g(x)}(p)$ and $f_{0}(x)=E_{g(x)}^{-1}(c)$. Given messages $a, b$ such that $f_{1}(a)=f_{0}(b), g(a)$ and $g(b)$ are a pair of keys with the desired property (i.e., $E_{g(b)}\left(E_{g(0 ;}(p)\right)=c$ ). To find a collision between $f_{1}$ and $f_{0}$, define the function $f$ as follows:

$$
f(x)=\left\{\begin{array}{l}
f_{1}(x) \text { if a particular bit of } x \text { is set } \\
f_{0}(x) \text { otherwise }
\end{array}\right.
$$

The particular bit that is used to choose between $f_{1}$ and $f_{0}$ is called the decision bit.

If DES is random, then we can expect collisions found in $f$ to be collisions between $f_{1}$ and $f_{0}$ about half of the time. This increases the expected work factor from $2^{36}$ in the singleDES collision case to $2^{37}$ in this case. 


\section{Four-Key Mapping}

The double-encryption collision finding above can be applied directly to the problem discussed in Section 2 of finding a set of permutations which map $p$ to $c$. However, we improved upon this approach by searching for four keys rather than two. We chose different functions $f_{1}$ and $f_{0}$ :

$$
f_{1}(x)=E_{h(x)}\left(E_{g(x)}(p)\right) \quad \text { and } \quad f_{0}(x)=E_{h(x)}^{-1}\left(E_{g(x)}^{-1}(c)\right)
$$

where functions $g$ and $h$ produce keys from messages, and the ordered pair $(g(x), h(x))$ is distinct for all $x \in M$. This approach doubles the number of encryptions which must be performed at each step of collision finding, but it eliminates the possibility of pseudocollisions. The expected number of steps required to find a collision in $f$ in this case is on the order of $\sqrt{|M|}=2^{32}$. To compare this running time to the two-key mapping above, we should take into account that fact that this approach requires two DES operations at each step instead of one. Also, only about half of the collisions in $f$ are collisions between $f_{1}$ and $f_{0}$. Thus, assuming that DES is random, the work factor in finding four keys with the required property is about $2^{34}$, which is eight times faster than finding a two-key mapping. The speed-up may be less than a factor of eight if the method of distinguished points is used for finding two-key mappings.

\section{Further Details on the Cycling Experiments}

In the cycling experiments, four-key mappings were sought as described in section 3 using the functions $f, f_{1}$, and $f_{0}$ in equations (1) and (2). The functions $g$ and $h$ in equation (2) were selected for ease of implementation. In the DES document [3], keys are represented in 64 bits with every eighth bit (bits $8,16, \ldots, 64$ ) a parity bit, ${ }^{1}$ leaving 56 independent bits. The function $g$ produces a key from a message by converting every eighth bit into a parity bit. Function $h$ produces a key from a message by shifting the message left one bit, and then converting every eighth bit into a parity bit. Note that the ordered pair $(g(x), h(x))$ is distinct for all $x \in M$ so that there is no possibility of pseudo-collisions.

As a test, a four-key mapping was sought for $p=c=0$. This value of $c$ is not one of the 50 randomly-selected values which contribute to the argument in section 2 . Using bit number 30 as the decision bit and an initial message $x_{0}=0123456789$ ABCDEF (hexadecimal) yielded a collision between $f_{1}$ and $f_{0}$ with the following results:

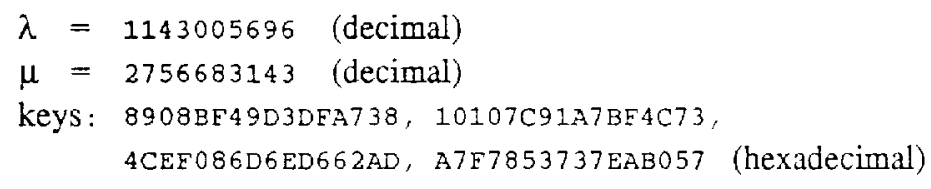

The results for the 50 random values of $c$ are given in the Appendix. There were no additional values of $c$ which were tried. This is important because failure for some values of $c$ would greatly diminish the confidence in the conclusions drawn in section 2 .

\footnotetext{
${ }^{2}$ In the DES document [3], bits of a message are numbered from 1 to 64 starting from the leftmost bit.
} 
These experiments were conducted over a four-month period using the background cycles on a set of workstations. The average number of workstations in use over the four-month period was about ten, and in the end, more than $10^{12}$ DES operations were performed.

\section{Conclusive Proof that DES is not Closed}

In an as yet unpublished paper, Don Coppersmith described his latest work on finding a lower bound on the size of the subgroup, $G$, generated by the DES permutations [1]. $\mathrm{He}$ takes advantage of special properties of $E_{0}$ and $E_{1}$ (DES encryption with the all 0 's and all 1's keys).

In earlier work [2], Coppersmith explained that the permutation $E_{1} E_{0}$ contains short cycles (of size about $2^{32}$ ). This makes it practical to find the length of the cycle produced by repeatedly applying $E_{1} E_{0}$ to some starting message. Each of these cycle lengths must divide the order of $E_{1} E_{0}$. Therefore, the least common multiple of the cycle lengths for various starting messages is a lower bound on the order of $E_{1} E_{0}$. Also, the order of $E_{1} E_{0}$ divides the size of $G$. This makes is possible to get a lower bound on the size of $G$.

Coppersmith found the cycle lengths for 33 messages which proved that the size of $G$ is at least $10^{277}$. We have found the cycle lengths for 295 additional messages (see Table 2 in the Appendix). Combining our results 'xith Coppersmith's yields a lower bound on the size of the subgroup generated by the DES permutations of $1.94 \times 10^{2499}$. This is greater than the number of DES permutations, which proves that DES is not closed. Also, meetin-the-middle attacks on DES which would exploit a small subgroup [4] are not feasible.

It is interesting to note that in the course of investigating the cycle structure of weak and semi-weak DES keys in 1986 [5], Moore and Simmons published 5 cycle lengths from which one could have concluded that $G$ has at least $2^{146}$ elements and that DES is not closed.

\section{Conclusion}

We have given probabilistic evidence as well as conclusive proof that DES is not a group. Furthermore, the subgroup generated by the DES permutations is more than large enough to prevent any meet-in-the-middle attacks which would exploit a small subgroup.

\section{Acknowledgement}

We would like to thank Alan Whitton for providing a large portion of our computing resources. 


\section{References}

1. D. Coppersmith, "In Defense of DES", personal communication, July 1992 (This work was also described briefly in a posting to sci.crypt on Usenet News, 1992 May 18).

2. D. Coppersmith, "The Real Reason for Rivest's Phenomenon", Advances in Cryptology - Crypto '85 Proceedings, Springer-Verlag, New York, pp. 535-536.

3. Dato Encryption Standord, Federal Infornation Processing Standards Publication 46, National Bureau of Standards, U.S. Department of Commerce, Washington, DC (1977 Jan. 15).

4. B.S. Kaliski, R.L. Rivest, and A.T. Sherman, "Is the Data Encryption Standard a Group? (Results of Cycling Experiments on DES)", Journal of Cryptology, vol. 1 (1988), no. 1, pp. 3-36.

5. J.H. Moore and G.J. Simmons, "Cycle Structure of the DES with Weak and Semi-weak Keys", Advances in Cryptology - Crypto' 86 Proceedings, Springer-Verlag, New York, pp. 9-32.

6. H. Morita, K. Ohta, and S. Miyaguchi, "A Switching Closure Test to Analyze Cryptosystems", Advances in Cryptology - Crypro' 91 Proceedings, Springer-Verlag, New York, pp. 183-193.

7. J.-J. Quisquater and J.-P. Delescaille, "How easy is collision search? Application to DES", Advances in Cryptology - Eurocrypt 89 Proceedings, Springer-Verlag, New York, pp. 429-434.

8. J.-J. Quisquater and J.-P. Delescaille, "How easy is collision search. New results and applications to DES", Advances in Cryptology - Crypto ' 89 Proceedings, Springer-Verlag, New York, pp. 408-413.

9. R. Sedgewick, T.G. Szymanski, and A.C. Yao, "The complexity of finding cycles in periodic functions", Siam Journal on Computing, vol. 11 (1982), no. 2, pp. 376-390.

\section{Appendix: Results of Cycling}

For each of 50 randomly selected messages $c$, Table 1 shows four DES keys $i, j, k$, and $l$ such that $E_{\mathfrak{d}}\left(E_{k}\left(E_{j}\left(E_{i}(0)\right)\right)\right)=c$. In each case, the initial message $x_{0}=0123456789 \mathrm{ABCDEF}$ was used. The DES keys in the table include eight parity bits as defined in the DES document [3]. The table also shows information from the collision search including the decision bit, the leader length $\lambda$. and the cycle length $\mu$. All quantities are shown in hexadecimal except the decision bit, $\lambda$, and $\mu$ which are shown in decimal.

Table 2 lists the cycle lengths obtained by applying the $E_{1} E_{0}$ permutation to various messages. 


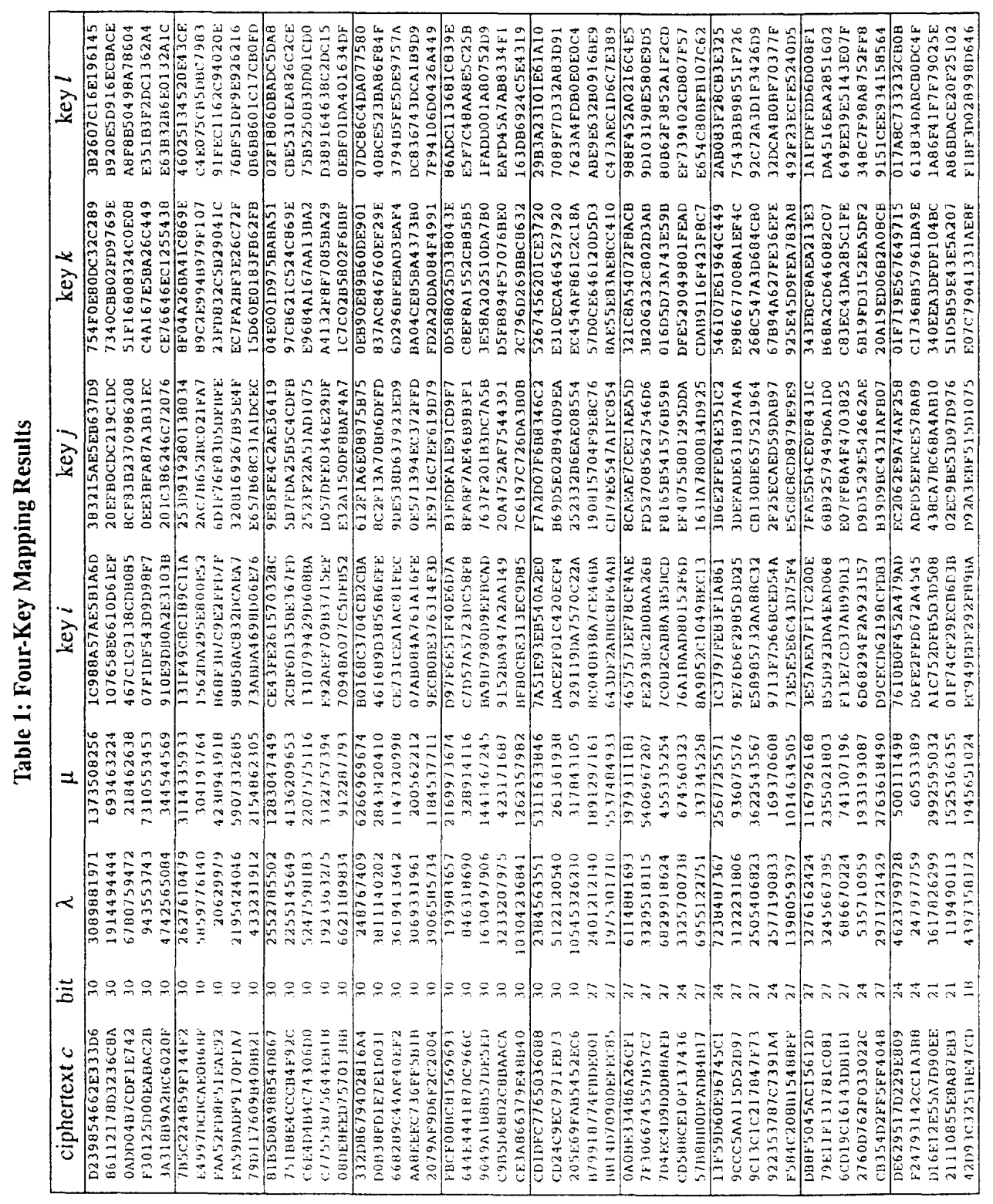




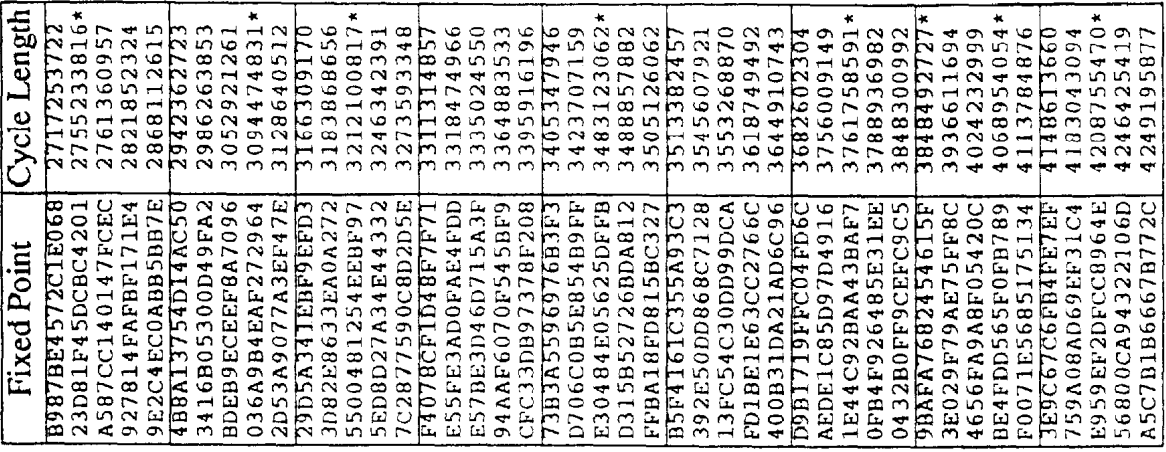

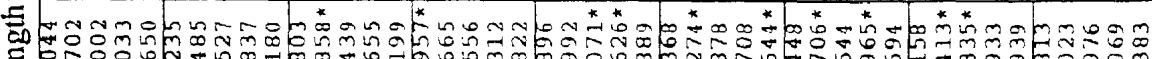

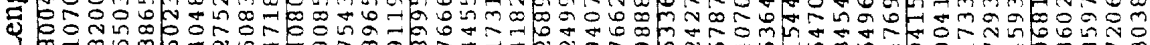
ש

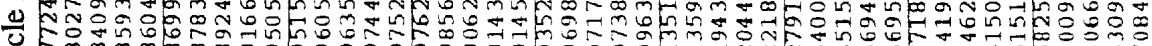
ऐ 염

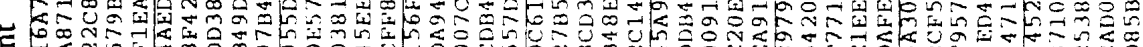

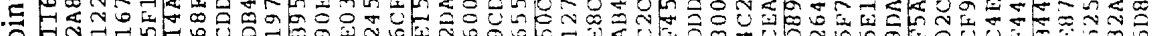

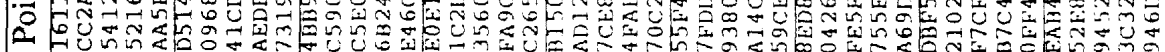

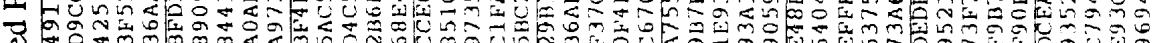

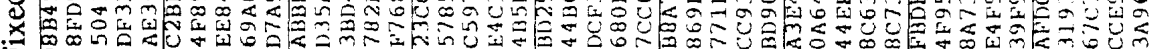

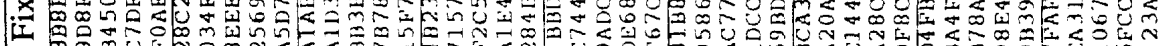

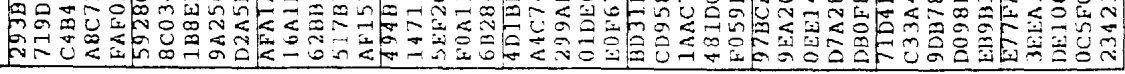

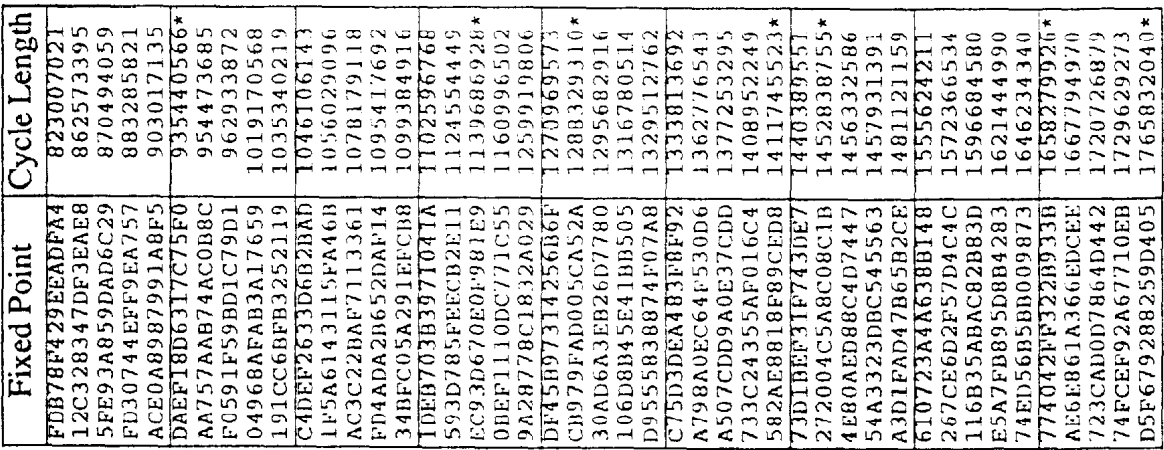

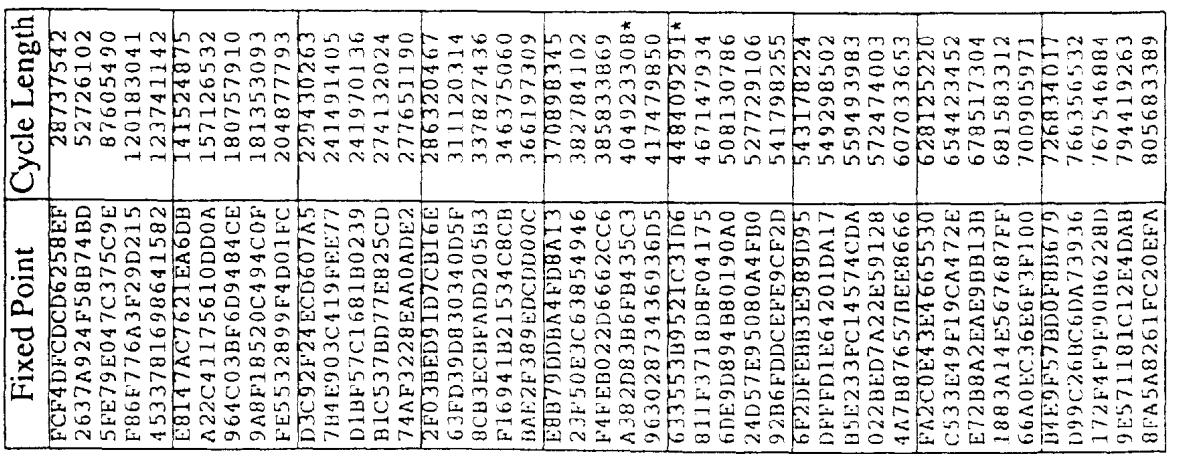



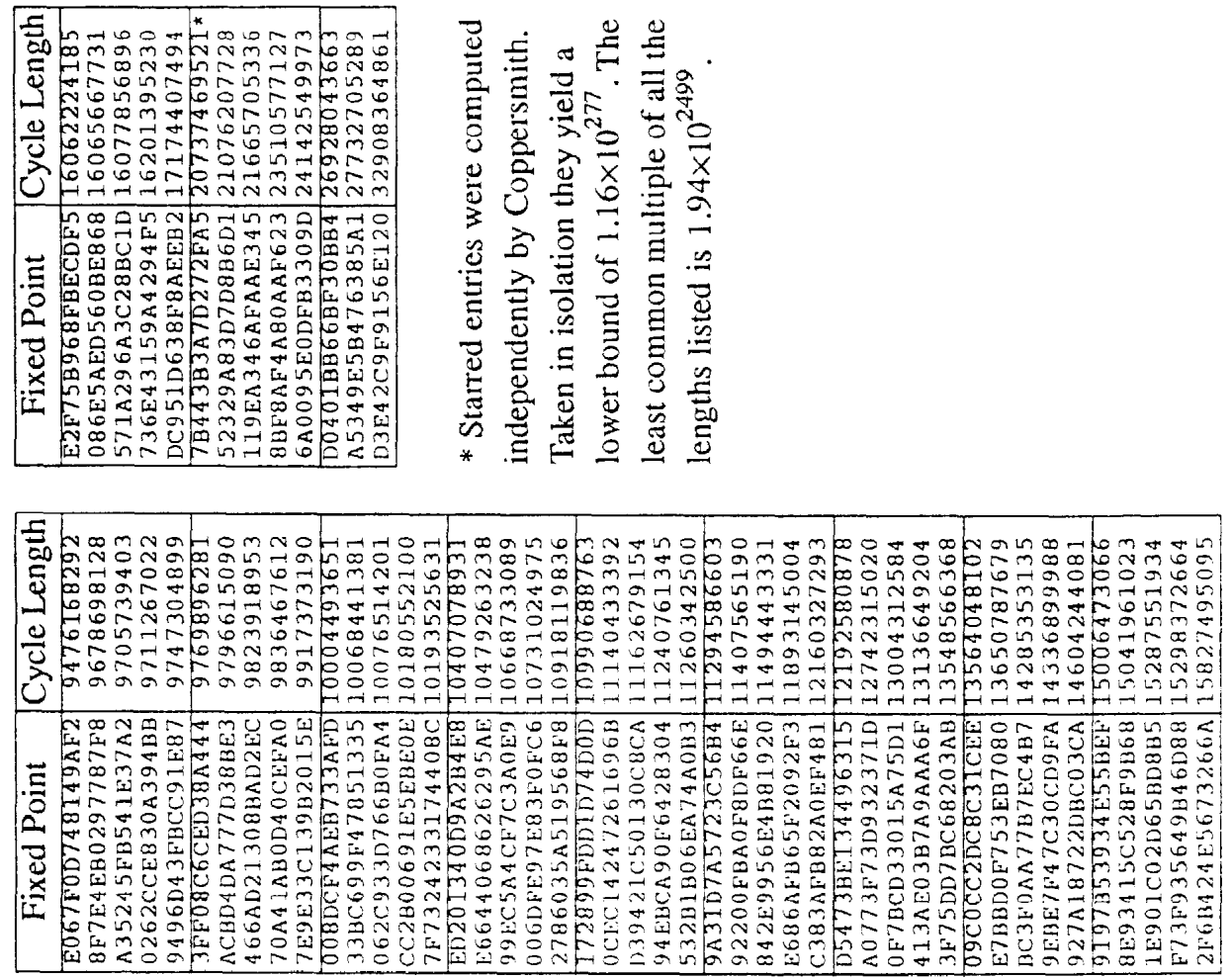

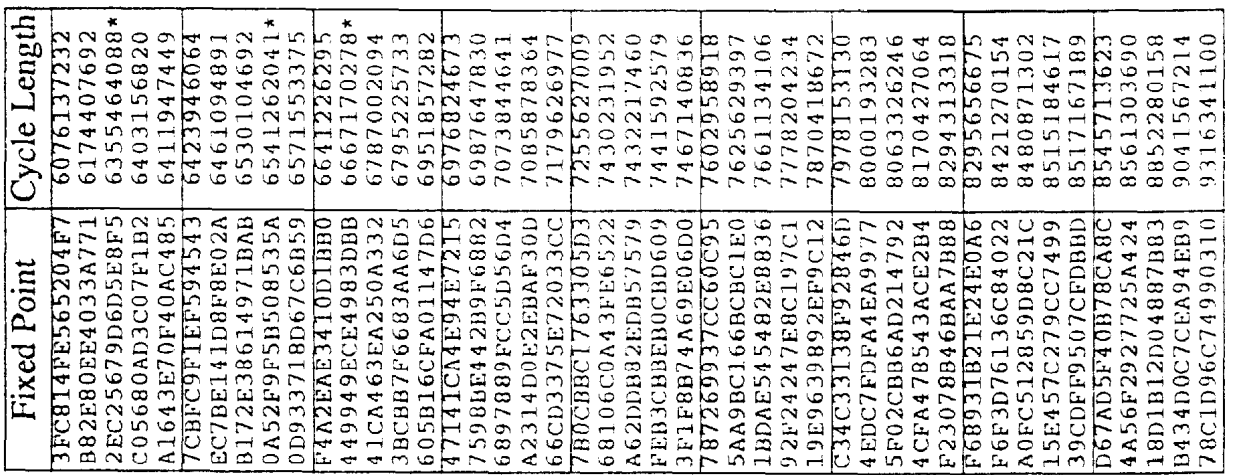

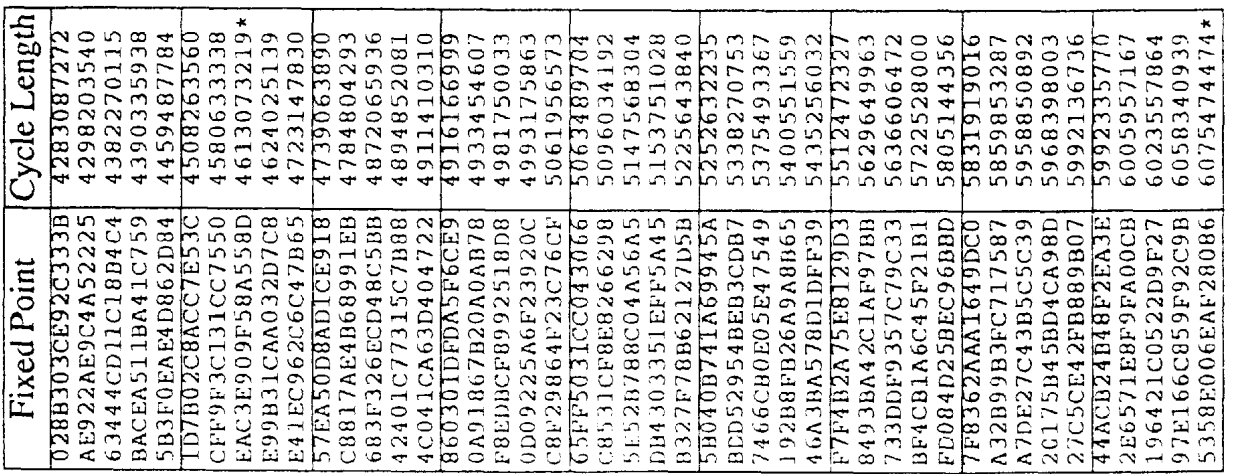

